\title{
Clinical characteristics of adult patients with systemic vasculitis: Data of 1348 patients from a single center
}

\author{
Jing $\mathrm{Li}^{1}$, Wenjie Zheng ${ }^{1}$, Yunjiao Yang ${ }^{1}$, Jiuliang Zhao ${ }^{1}$, Mengtao $\mathrm{Li}^{1}$, Yanhong Wang ${ }^{2}$, Xinping Tian ${ }^{1, *}$, Xiaofeng Zeng ${ }^{1, *}$
}

${ }^{1}$ Department of Rheumatology and Clinical Immunology, Peking Union Medical College and Chinese Academy of Medical Sciences; National Clinical Research Center for Dermatologic and Immunologic Diseases (NCRC-DID), Ministry of Science \& Technology; State Key Laboratory of Complex Severe and Rare Diseases, Peking Union Medical College Hospital (PUMCH), Key Laboratory of Rheumatology and Clinical Immunology, Ministry of Education, Beijing 100730, China

${ }^{2}$ Department of Epidemiology and Biostatistics, Institute of Basic Medical Sciences, Chinese Academy of Medical Sciences, School of Basic Medicine, Peking Union Medical College, Beijing 100005, China

Abstract

Received May 5, 2021 accepted July 21, 2021

Objective: To investigate the clinical characteristics of patients with systemic vasculitis in China by analyzing the data from a nationwide registry database, the Chinese Registry for Systemic Vasculitis (CRSV).

Methods: The demographic data, clinical presentations, image and laboratory test results, disease activity assessment, treatment, and outcome of patients enrolled with the confirmed diagnosis of systemic vasculitis, including Takayasu's arteritis (TAK), ANCA-associated vasculitis (AAV), Behcet's syndrome (BD), Polyangitis nodosa (PAN), and unclassified systemic vasculitis (USV), were collected since July 2013 in the CRSV. The data during July 2013 and February 28 of 2020 were retrieved and analyzed.

Results: Up to February 2020, 3852 patients in total were registered in the CRSV. In Peking Union Medical College Hospital (PUMCH), 1348 patients were registered, including 730 patients with TAK, 343 with Behcet's disease (BD), 191 with AAV, 53 with USV, and 31 with PAN. More male patients were found in PAN (64.5\%), BD (59.8\%), and AAV (52.4\%), while TAK was more prevalent in female patients (85.9\%). The average age at disease diagnosis was 29.6 in TAK, 33.2 in BD, 28.6 in PAN, 39.4 in USV, and 48.1 in AAV. A significant difference was found between age at diagnosis of TAK and AAV. Gender comparisons revealed that the age at diagnosis of male patients with AAV was older than female patients. Hypertension $(\mathbf{5 7 . 3 \% )}$ and stroke $(\mathbf{1 7 . 5 \% )}$ were found to be more common in male patients with TAK, vascular involvement $(27.3 \%)$ was found to be more common in male patients with BD, while neurological involvement $(29.0 \%)$ was found to be more common in male patients with AAV than in females.

Conclusion: This registry is the first in China and provides rich information about the clinical characteristics of Chinese patients with systemic vasculitis. There are significant differences in clinical presentations between different categories of systemic vasculitis.

Keywords

ANCA-associated vasculitis • Behcet's disease • clinical characteristics • polyarteritis nodosa • registry study • Takayasu arteritis - unclassified systemic vasculitis

\footnotetext{
Address for correspondence:

*Xinping Tian / Xiaofeng Zeng, Department of Rheumatology and Clinical Immunology, Peking Union Medical College and Chinese Academy of Medical Sciences; National Clinical Research Center for Dermatologic and Immunologic Diseases (NCRC-DID), Ministry of Science \& Technology; State Key Laboratory of Complex Severe and Rare Diseases, Peking Union Medical College Hospital (PUMCH), Key Laboratory of Rheumatology and Clinical Immunology, Ministry of Education, Beijing 100730, China. E-mail: tianxp6@126.com.
} 


\section{Introduction}

Systemic vasculitis is a group of uncommon rheumatic diseases characterized by multiple system involvement and complicated clinical manifestations. The diagnosis and treatment of systemic vasculitis has been one of the biggest challenges in rheumatology. Better understanding of the clinical characteristics, patients' response to treatment, and factors related to short- and long-term prognosis is important to provide the basis for future research. Therefore, building up a national prospective cohort of patients with systemic vasculitis is an important way to understand in-depth the clinical profiles and outcome features of patients with systemic vasculitis. In this study, we introduce the development of a nationwide registry database, the Chinese Registry for Systemic Vasculitis (CRSV), and summarize the clinical features of Chinese patients with systemic vasculitis.

In order to understand the disease characteristics of systemic vasculitis in China, we developed a nationwide registry platform, called the CRSV, which is based on the Chinese Rheumatism Data Center (CRDC) platform. The CRDC was established originally by Peking Union Medical College Hospital (PUMCH) in 2011 supported by the National Health and Family Planning Commission. ${ }^{[1]}$ CRDC is a platform for patient cohort development and clinical research. More than half of the Chinese rheumatologists affiliated to 282 medical institutions participated in the registry. The CRSV registry was initiated in July 2013. Twenty-one tertiary medical centers participated in the registry. The goal of this registry is to better understand the epidemiology, clinical characteristics, and the short- and long-term outcomes of Chinese patients with systemic vasculitis. Patients with 5 types of systemic vasculitis were registered in the CRSV, including Takayasu's arteritis (TAK), Behcet's disease (BD), ANCA-associated vasculitis (AAV), polyarteritis nodosa (PAN), and unclassified systemic vasculitis (USV). After being registered, patients are followed up regularly.

\section{Methods}

\section{Data Collection}

Patients in the CRSV must meet the classification criteria for each individual type of vasculitis. The data collected include demographic information, related medical histories, clinical manifestations, laboratory test results, imaging findings, disease activity assessment, treatment regimen, intervention or surgical procedures, adverse events related to medications, and complications associated with procedures and interventions as well as the outcomes. The core dataset of TAK is shown in Table 1 as an example. Scheduled visits

Table 1: The clinical characteristics of patients with Takayasu's arteritis registered by PUMCH in CRSV

\begin{tabular}{|c|c|c|c|c|}
\hline & Total & Male & Female & P-value \\
\hline Number of Patients & 730 & $103(14.1 \%)$ & $627(85.9 \%)$ & \\
\hline Age at diagnosis & $29.6 \pm 10.8(3.3,73.8)$ & $31.1 \pm 12.6(3.3,73.8)$ & $29.3 \pm 10.4(11.1,66.1)$ & 0.18 \\
\hline Age at registry & $32.3 \pm 10.8(13.0,74.0)$ & $33.6 \pm 12.1(14.4,74.0)$ & $32.1 \pm 10.6(13.0,66.1)$ & 0.63 \\
\hline Disease duration on registry(years) & $2.5 \pm 4.8(0,41.0)$ & $2.3 \pm 5.4(0,27.7)$ & $2.6 \pm 4.7(0,41.0)$ & 0.18 \\
\hline \multicolumn{5}{|l|}{ Laboratory findings } \\
\hline White blood cells $\left(\times 10^{9} / \mathrm{L}\right)$ & $8.7 \pm 3.4$ & $8.8 \pm 3.0$ & $8.7 \pm 3.4$ & 0.68 \\
\hline Hemoglobin (g/L) & $123.3 \pm 18.1$ & $141.5 \pm 16.1$ & $120.4 \pm 16.7$ & $<0.001^{* * *}$ \\
\hline Platelet $\left(\times 10^{9} / \mathrm{L}\right)$ & $327.5 \pm 839.0$ & $271.5 \pm 97.4$ & $336.4 \pm 901.8$ & 0.50 \\
\hline ESR ( $1^{\text {sthour }}$ ) & $25.2 \pm 27.9$ & $21.3 \pm 27.9$ & $25.8 \pm 27.9$ & 0.55 \\
\hline $\mathrm{CRP}(\mathrm{mg} / \mathrm{L})$ & $18.2 \pm 31.0$ & $20.1 \pm 30.1$ & $17.9 \pm 31.2$ & 0.15 \\
\hline \multicolumn{5}{|l|}{ Clinical evaluations } \\
\hline BVAS & $9.6 \pm 5.4$ & $8.4 \pm 5.2$ & $9.8 \pm 5.4$ & $0.02^{*}$ \\
\hline VDI & $3.0 \pm 1.7$ & $3.0 \pm 1.7$ & $3.0 \pm 1.8$ & 0.95 \\
\hline \multicolumn{5}{|l|}{ Clinical features } \\
\hline Hypertension & $331(45.3 \%)$ & $59(57.3 \%)$ & $272(43.4 \%)$ & $<0.01 * *$ \\
\hline Fatigue & $307(42.0 \%)$ & $33(42.3 \%)$ & $249(48,8 \%)$ & 0.14 \\
\hline Fever ( $>38$ centigrade) & $185(25.3 \%)$ & $20(25.6 \%)$ & $146(28.6 \%)$ & 0.39 \\
\hline Arthralgia or arthritis & $142(19.5 \%)$ & $10(12.8 \%)$ & $98(19.2 \%)$ & 0.12 \\
\hline Weight loss & $128(17.5 \%)$ & $19(24.4 \%)$ & 99 (19.4\%) & 0.50 \\
\hline \multicolumn{5}{|l|}{ Sign and symptoms of peripheral vessels } \\
\hline Vessel bruits in neck area & $534(73.2 \%)$ & $55(53.4 \%)$ & $479(76.4 \%)$ & $<0.001^{* * *}$ \\
\hline Asymmetric blood pressure in bilateral limbs & 452 (61.9\%) & $52(50.5 \%)$ & $400(63.8 \%)$ & $0.01 *$ \\
\hline
\end{tabular}


Table 1: Continued

\begin{tabular}{|c|c|c|c|c|}
\hline & Total & Male & Female & $P$-value \\
\hline Decreased limb arterial pulsation & $437(59.8 \%)$ & $57(55.3 \%)$ & $380(60.6 \%)$ & 0.31 \\
\hline Decreased radial artery pulsation & $388(53.2 \%)$ & $47(45.6 \%)$ & $341(54.4 \%)$ & 0.10 \\
\hline Intermittent claudication of limbs & $336(46.0 \%)$ & $36(35.0 \%)$ & $300(47.8 \%)$ & $0.02 *$ \\
\hline Intermittent claudication of tongue or mandible & $58(7.9 \%)$ & $6(5.8 \%)$ & $52(8.3 \%)$ & 0.39 \\
\hline Carotidynia & $195(26.7 \%)$ & $18(17.5 \%)$ & $177(28.2 \%)$ & $0.02 *$ \\
\hline Cardiac abnormalities & $391(53.6 \%)$ & $52(50.5 \%)$ & $339(54.1 \%)$ & 0.50 \\
\hline Aortic insufficiency & $250(34.2 \%)$ & $27(26.2 \%)$ & $223(35.6 \%)$ & 0.06 \\
\hline Heart murmur & $107(14.7 \%)$ & 15 (14.6\%) & $92(14.7 \%)$ & 0.98 \\
\hline Pericardial effusion & $103(14.1 \%)$ & $9(8.7 \%)$ & $94(15.0 \%)$ & 0.09 \\
\hline Angina & $52(7.1 \%)$ & $10(9.7 \%)$ & $42(6.7 \%)$ & 0.27 \\
\hline Congestive heart failure & $49(6.7 \%)$ & $7(6.8 \%)$ & $42(6.7 \%)$ & 0.97 \\
\hline Myocardial infarct & $18(2.5 \%)$ & $4(3.9 \%)$ & $14(2.2 \%)$ & $0.30^{\mathrm{F}}$ \\
\hline Cardiomyopathy & $16(2.2 \%)$ & $1(1.0 \%)$ & $15(2.4 \%)$ & $0.71^{\mathrm{F}}$ \\
\hline \multicolumn{5}{|l|}{ Neurological involvement } \\
\hline Headache & $257(35.2 \%)$ & $26(26.2 \%)$ & $231(36.8 \%)$ & $0.02^{*}$ \\
\hline Dizziness & $271(37.1 \%)$ & $27(28.2 \%)$ & $244(38.9 \%)$ & $0.01^{*}$ \\
\hline Syncope & 91 (12.5\%) & $8(7.8 \%)$ & $83(13.2 \%)$ & 0.12 \\
\hline Stroke & $62(8.5 \%)$ & $18(17.5 \%)$ & $44(7.0 \%)$ & $<0.001^{* * *}$ \\
\hline TIA & $54(7.4 \%)$ & $9(8.7 \%)$ & $45(7.2 \%)$ & 0.58 \\
\hline Cranial nerve injury & $3(0.4 \%)$ & $1(1.0 \%)$ & $2(0.3 \%)$ & $0.37^{F}$ \\
\hline \multicolumn{5}{|l|}{ Renal abnormalities } \\
\hline Abnormalities in renal arteries & $242(33.2 \%)$ & $35(34.0 \%)$ & $207(33.0 \%)$ & 0.85 \\
\hline Urine protein over $0.2 \mathrm{~g}$ in 24 hours & $13(1.8 \%)$ & $1(1.0 \%)$ & $12(1.9 \%)$ & $1.00^{\mathrm{F}}$ \\
\hline Cutaneous and mucosal lesions & $124(17.0 \%)$ & $16(15.5 \%)$ & $108(17.2 \%)$ & 0.67 \\
\hline Oral ulceration & $73(10.0 \%)$ & $12(11.6 \%)$ & $61(9.7 \%)$ & 0.55 \\
\hline Erythema nodosum & $38(5.2 \%)$ & $3(2.9 \%)$ & $35(5.6 \%)$ & 0.26 \\
\hline Cutaneous vasculitis & $22(3.0 \%)$ & $1(1.0 \%)$ & $21(3.2 \%)$ & $0.35^{\mathrm{F}}$ \\
\hline Skin ulceration & $4(0.5 \%)$ & $1(1.0 \%)$ & $3(0.5 \%)$ & $0.46^{\mathrm{F}}$ \\
\hline Skin Purpura & $7(1.0 \%)$ & $1(1.0 \%)$ & $6(1.0 \%)$ & $1.00^{\mathrm{F}}$ \\
\hline Ocular involvement & $112(15.3 \%)$ & $7(6.8 \%)$ & $105(16.7 \%)$ & $<0.01^{* *}$ \\
\hline Amaurosis & $85(11.6 \%)$ & $6(5.8 \%)$ & $79(12.6 \%)$ & $0.047^{*}$ \\
\hline Retinal vasculitis/ischemic retinitis & $36(4.9 \%)$ & $2(1.9 \%)$ & $34(5.4 \%)$ & 0.13 \\
\hline Diplopia & $33(4.5 \%)$ & $1(1.0 \%)$ & $32(5.1 \%)$ & $0.07^{\mathrm{F}}$ \\
\hline Optic atrophy & $1(0.1 \%)$ & 0 & $1(0.2 \%)$ & $1.00^{\mathrm{F}}$ \\
\hline Pulmonary abnormalities & $72(9.1 \%)$ & $12(11.7 \%)$ & $60(9.6 \%)$ & 0.51 \\
\hline Pulmonary arterial hypertension & $30(4.1 \%)$ & $3(2.9 \%)$ & $27(4.3 \%)$ & $0.79^{\mathrm{F}}$ \\
\hline Pulmonary infarction & $14(1.9 \%)$ & $2(1.9 \%)$ & $12(1.9 \%)$ & $1.00^{\mathrm{F}}$ \\
\hline Gastrointestinal tract abnormalities & $29(4.0 \%)$ & $4(3.9 \%)$ & $25(4.0 \%)$ & $1.00^{\mathrm{F}}$ \\
\hline Mesenteric ischemia & $14(1.9 \%)$ & $2(1.9 \%)$ & $12(1.9 \%)$ & $1.00^{\mathrm{F}}$ \\
\hline Intestinal perforation/infarction & $2(0.3 \%)$ & 0 & $2(0.3 \%)$ & $1.00^{\mathrm{F}}$ \\
\hline Angioplasty & $199(27.3 \%)$ & $34(33.0 \%)$ & $165(26.3 \%)$ & 0.16 \\
\hline Venous thrombosis in vessels & $36(4.9 \%)$ & $10(9.7 \%)$ & $26(4.1 \%)$ & $0.02^{*}$ \\
\hline Collapse of vertebra/Osteoporosis & $7(1.0 \%)$ & 0 & $7(1.1 \%)$ & $0.60^{\mathrm{F}}$ \\
\hline Aseptic necrosis & $4(0.5 \%)$ & 0 & $4(0.6 \%)$ & $1.00^{\mathrm{F}}$ \\
\hline
\end{tabular}

${ }^{*} P<0.05 ;{ }^{*} P<0.01 ;{ }^{* *} P<0.001$. ${ }^{\mathrm{F}}$ Fisher's exact test.

Abbreviation: PUMCH, Peking Union Medical College Hospital. CRSV, Chinese Registry for Systemic Vasculitis. ESR, erythrocyte sediment rate. CRP, c-reactive protein. BVAS, Birmingham vasculitis activity score. VDI, vasculitis damage index. TIA, transient ischemic attack.

Legend: The clinical characteristics of patients with Takayasu's arteritis registered by Peking Union Medical College Hospital (PUMCH) in Chinese Registry for Systemic Vasculitis (CRSV) database were summarized. The comparisons between male and female patients were made. The data was up to February 28 of 2020. 
are conducted in accordance with disease categories and patients' disease status with at least 1 visit every 6 months to evaluate the disease activity if the patient is in a stable condition in the present follow-up visit. Emergency contact messages were provided to patients and could be transferred to the caring rheumatologist within $24 \mathrm{~h}$.

\section{Classification Criteria}

All patients registered in the CRSV were diagnosed with primary systemic vasculitis; vasculitis secondary to infection or malignancy were excluded. Patients with $\mathrm{TAK}^{\left[{ }^{[2]}\right.} \mathrm{PAN},{ }^{[3]}$ granulomatosis with polyangiitis (formerly called Wegener granulomatosis), ${ }^{[4]}$ and eosinophilic granulomatosis with polyangiitis (formerly called Churg-Strauss syndrome) ${ }^{[5]}$ were diagnosed according to the 1990 ACR classification criteria, respectively. Patients with microscopic polyangiitis were diagnosed according to the 2012 Chapel Hill consensus, ${ }^{\left[{ }^{[6]}\right.}$ and patients with BD were diagnosed according to the 2013 international criteria for $\mathrm{BD} .{ }^{[7]}$ Other patients with systemic vasculitis who could not be classified into the above six types of disease and did not fulfill the classification criteria of giant cell arteritis ${ }^{[8]}$ were registered as USV.

\section{Data Quality Control}

The CRSV is an electronic data registry system, and measures have been applied to control the quality of data input. Auto-checking mechanism and data auditing system are applied as the essential data quality control measure. The second-level quality control is logical checking to make sure that the data entered into the system are not logically contradictory. We also built-up a predefined treatment regimen menu to facilitate treatment data entry. Finally, quality inspection and feedback are conducted by a professional third-party quality control team.

\section{Ethical and Legal Consideration}

Informed consent was obtained for each individual patient who was registered in the CRSV registry in full compliance with Chinese laws and regulations. The patients' private information is stored in an encrypted manner, and only the physicians who are taking care of the patients and the patients themselves are eligible to access these data. This registry was approved by the Institutional Review Board of PUMCH (S-478).

\section{Data Analysis}

Since the detailed information of individual patients from other centers could not be identified due to the privacy management rule of CRDC, we included only the data from $\mathrm{PUMCH}$ single center for analysis. The demographic data and clinical features were summarized herein. Furthermore, comparisons between genders and age were made.

\section{Statistical Analysis}

Since all data were not distributed in a normal pattern, we described the numerical variables as medians (Q1, Q3), and the categorical variables as numbers (percentage). Comparisons between different populations were made using Chi-square tests for categorical data. Fisher's exact tests were conducted when the expected frequencies were $<5$. A two-sided $P$-value $<0.05$ was considered to be statistically significant. Analysis was performed with the SPSS software (version 19.0; IBM SPSS statistics, Armonk, New York, USA).

\section{Results}

Up to February 28, 2018, 2051 patients with systemic vasculitis were registered into the CRSV database, including 778 patients with BD, 737 patients with TAK, 339 patients with AAV, 167 patients with USV, and 30 patients with PAN. After 2 years (up to February 28, 2020), 3852 patients with systemic vasculitis were registered into the CRSV database in total, including 1390 patients with BD, 1318 patients with TAK, 773 patients with AAV, 317 patients with USV, and 54 patients with PAN (Figure 1). A total of 1348 patients were registered by $\mathrm{PUMCH}$, including 730 patients with TAK, 343 patients with BD, 191 patients with AAV, 53 patients with USV, and 31 patients with PAN. We summarized the gender distribution of these 1348 patients from 5 types of systemic vasculitis diseases, respectively, in Figure 2. The proportions of male patients were higher than females in PAN $(20 / 31$, $64.5 \%)$, BD (205/343, 59.8\%), and AAV (100/191, 52.4\%). On the contrary, the proportion of female patients was higher in TAK $(627 / 730,85.9 \%)$ and USV $(29 / 53,54.7 \%)$ patients than in males (Figure 2).

There was an uptrend in the age at disease diagnosis and registration for patients with TAK (29.6/32.3), BD (33.2/35.7), PAN (38.6/41.0), USV (39.4/40.6), and AAV (48.1/49.4) (Tables 1-4). As patients with BD usually have a long-term history of oral ulceration without a specific time of disease onset, their age on diagnosis during registration was not accurately required. Comparison between ages at diagnosis and registration of patients with TAK and AAV were statistically significantly different $(P<0.001)$.

The major clinical characteristics of TAK, BD, AAV, USV, and PAN are summarized in Tables 1-5. Vascular abnormalities including physical findings of vessel bruits (73.2\%), asymmetric blood pressure in bilateral limbs $(61.9 \%)$, limbs claudication (46.0\%), and carotidynia (26.7\%) were the most common clinical findings in TAK patients, and the constitutional symptoms were also common, such as fever $(25.3 \%)$ and weight loss (17.5\%). Heart (53.6\%) and kidney $(33.2 \%$, mainly as a consequence of involvement of renal artery) were the most commonly involved organs in TAK. Cutaneous and mucosal lesion (17.0\%), ocular 


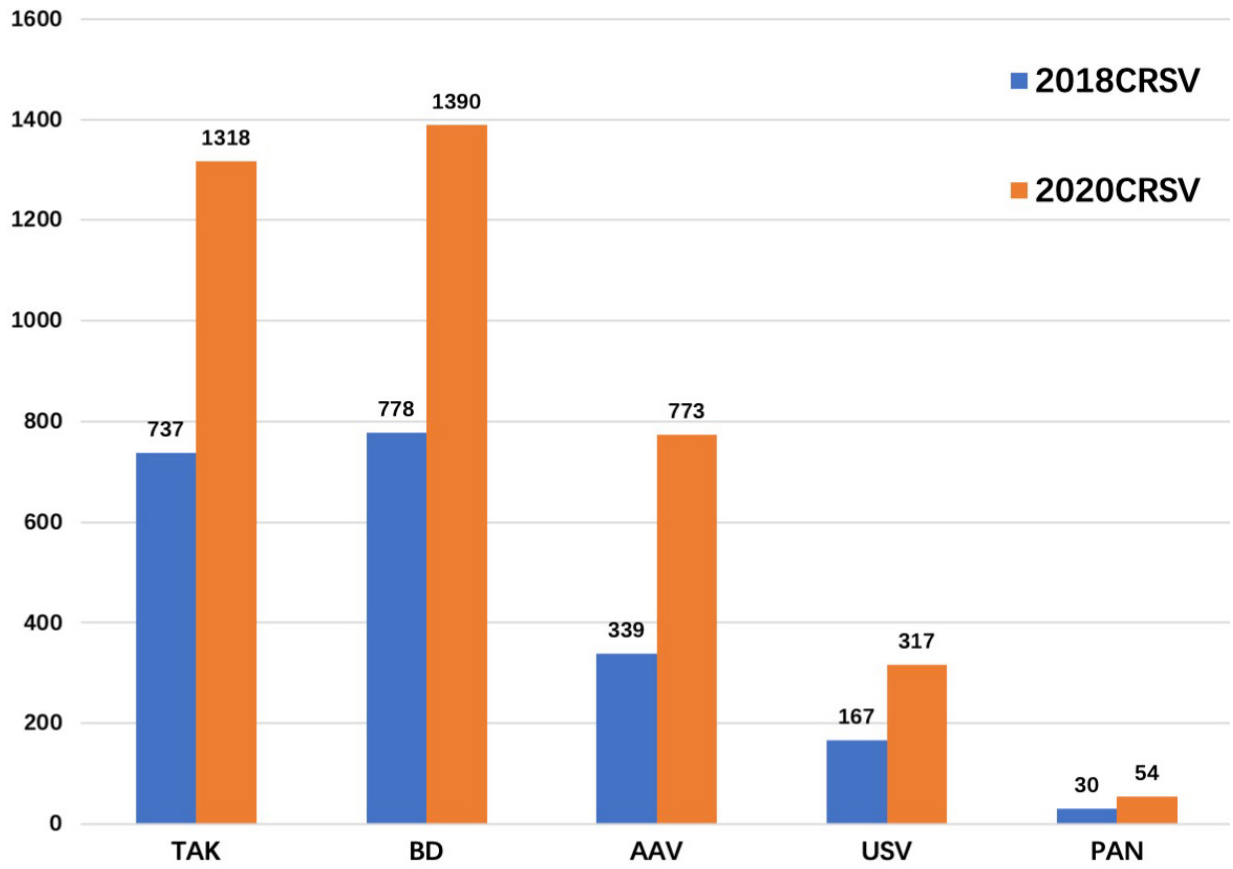

Figure 1: Patients with TAK, BD, AAV, USV, and PAN registered in the CRSV increased rapidly from 2018 to 2020. Up to February 28 of 2020 , compared with 2 years ago (up to February 28 of 2018), the patients with TAK, BD, AAV, USV and PAN registered in the CRSV nationwide increased rapidly. AAV, ANCA-associated vasculitis; $B D$, Behcet's disease; CRSV, Chinese Registry for Systemic Vasculitis; PAN, polyarteritis nodosa; TAK, Takayasu's arteritis; USV, unclassified systemic vasculitis.

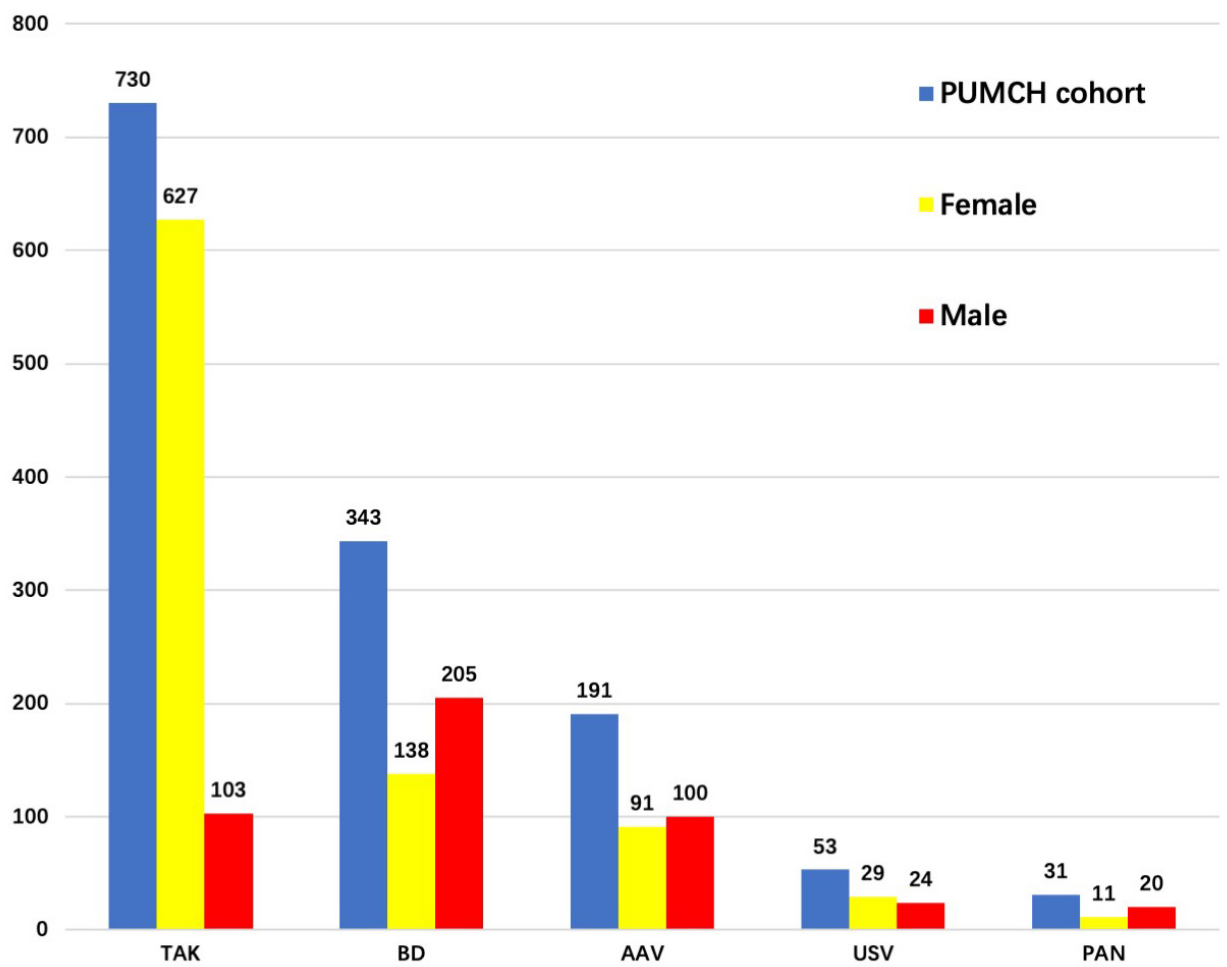

Figure 2: Patients TAK, BD, AAV, USV, and PAN registered in one center (PUMCH) with gender distributions. In a single center of the CRSV, $P U M C H$, the proportions of male patients were higher than female ones in BD, AAV, and PAN. On the contrary, the proportion of female patients was much higher in TAK patients than male ones. AAV, ANCA-associated vasculitis; BD, Behcet's disease; CRSV, Chinese Registry for Systemic Vasculitis; PUMCH, Peking Union Medical College Hospital; PAN, polyarteritis nodosa; TAK, Takayasu's arteritis; USV, unclassified systemic vasculitis. 
Original Article $\bullet$ DOI: $10.2478 /$ rir-2021-0014 $\cdot 2(2) \cdot 2021 \cdot 101-112$

Table 2: The clinical characteristics of patients with Behcet's disease registered by PUMCH in CRSV

\begin{tabular}{|c|c|c|c|c|}
\hline & Total & Male & Female & $P$-value \\
\hline Number of Patients & 343 & $205(59.8 \%)$ & $138(40.2 \%)$ & \\
\hline Age at diagnosis (years) & $33.2 \pm 10.3(11.6,59.6)$ & $33.1 \pm 10.0(11.6,58.6)$ & $33.4 \pm 10.6(11.8,59.6)$ & 0.80 \\
\hline Age at registration (years) & $35.7 \pm 10.7(14.2,69.2)$ & $35.4 \pm 10.5(14.2,69.2)$ & $36.3 \pm 11.0(15.7,64.3)$ & 0.47 \\
\hline Disease duration before registration (years) & $2.5 \pm 4.2(0,26.7)$ & $2.3 \pm 4.1(0,26.7)$ & $2.9 \pm 4.5(0,20.3)$ & 0.21 \\
\hline \multicolumn{5}{|l|}{ Laboratory results } \\
\hline White blood cells $\left(\times 10^{9} / \mathrm{L}\right)$ & $8.0 \pm 2.9$ & $8.5 \pm 2.8$ & $7.3 \pm 2.8$ & $<0.01^{* *}$ \\
\hline Hemoglobin (g/L) & $134.6 \pm 19.3$ & $142.0 \pm 18.1$ & $124.2 \pm 15.8$ & $<0.001^{* * *}$ \\
\hline Platelet $\left(\times 10^{9} / \mathrm{L}\right)$ & $265.4 \pm 88.7$ & $254.7 \pm 82.8$ & $279.7 \pm 94.6$ & 0.03 \\
\hline ESR ( $1^{\text {st }}$ hour $)$ & $20.1 \pm 21.7$ & $19.4 \pm 22.3$ & $21.1 \pm 20.8$ & 0.54 \\
\hline $\mathrm{CRP}(\mathrm{mg} / \mathrm{L})$ & $15.0 \pm 23.3$ & $16.3 \pm 22.2$ & $13.0 \pm 24.8$ & 0.29 \\
\hline \multicolumn{5}{|l|}{ Clinical manifestations } \\
\hline VDI & $1.5 \pm 1.1(0,6)$ & $1.6 \pm 1.1(0,6)$ & $1.4 \pm 1.0(0,6)$ & 0.30 \\
\hline \multicolumn{5}{|l|}{ Clinical manifestations } \\
\hline Fatigue & $178(51.9 \%)$ & $110(53.7 \%)$ & $68(49.3 \%)$ & 0.43 \\
\hline Fever $\left(\geq 38^{\circ} \mathrm{C}\right)$ & $100(29.2 \%)$ & $63(30.7 \%)$ & $37(26.8 \%)$ & 0.43 \\
\hline Arthralgia/arthritis & $66(19.2 \%)$ & $38(18.5 \%)$ & $28(20.3 \%)$ & 0.69 \\
\hline Weight loss & $57(16.6 \%)$ & $34(16.6 \%)$ & $23(16.7 \%)$ & 0.98 \\
\hline Cutaneous and mucosal lesions & $276(80.5 \%)$ & $161(78.5 \%)$ & $115(83.3 \%)$ & 0.27 \\
\hline Oral ulcer & $276(80.5 \%)$ & $162(79.0 \%)$ & $114(82.6 \%)$ & 0.41 \\
\hline Genital ulcer & $201(58.6 \%)$ & $104(50.7 \%)$ & $97(70.3 \%)$ & $<0.001^{* * *}$ \\
\hline Erythema nodosum & $121(35.3 \%)$ & $74(36.1 \%)$ & $47(34.1 \%)$ & 0.70 \\
\hline Pseudofolliculitis & $56(16.3 \%)$ & $42(20.5 \%)$ & $14(10.1 \%)$ & $0.01^{*}$ \\
\hline Papular pustule & $34(9.9 \%)$ & $24(11.7 \%)$ & $10(7.2 \%)$ & 0.18 \\
\hline Acne like rash & $30(8.7 \%)$ & $24(11.7 \%)$ & $6(4.3 \%)$ & $0.02 *$ \\
\hline Skin ulcer & $10(2.9 \%)$ & $8(3.9 \%)$ & $2(1.4 \%)$ & $0.33^{\mathrm{F}}$ \\
\hline Ocular involvement & $98(28.6 \%)$ & $63(30.7 \%)$ & $35(25.4 \%)$ & 0.28 \\
\hline Blurred vision & $69(20.1 \%)$ & $50(24.4 \%)$ & $19(13.8 \%)$ & $0.02 *$ \\
\hline Uveitis & $63(18.4 \%)$ & $47(22.9 \%)$ & $16(11.6 \%)$ & $<0.01^{* *}$ \\
\hline Diplopia & $61(17.8 \%)$ & $40(19.5 \%)$ & $21(15.2 \%)$ & 0.31 \\
\hline Retinal vasculitis & $15(4.4 \%)$ & $11(5.4 \%)$ & $4(2.9 \%)$ & 0.27 \\
\hline Visual loss & $14(4.1 \%)$ & $11(5.4 \%)$ & $3(2.2 \%)$ & 0.14 \\
\hline Cataract & $14(4.1 \%)$ & $12(5.9 \%)$ & 2 (1.4\%) & $0.04^{*}$ \\
\hline Pulmonary abnormalities & $25(7.3 \%)$ & $15(7.3 \%)$ & $10(7.2 \%)$ & 0.98 \\
\hline Abnormalities of heart & $24(7.0 \%)$ & $17(8.3 \%)$ & $7(5.1 \%)$ & 0.25 \\
\hline Gastrointestinal tract involvement & $74(21.6 \%)$ & $40(19.5 \%)$ & $34(24.6 \%)$ & 0.26 \\
\hline Esophageal ulcer & $20(5.8 \%)$ & $12(5.9 \%)$ & $8(5.8 \%)$ & 0.98 \\
\hline Ulcers of colon & $17(5.0 \%)$ & $9(4.4 \%)$ & $8(5.8 \%)$ & 0.56 \\
\hline Renal abnormalities & $6(1.7 \%)$ & $3(1.5 \%)$ & $3(2.2 \%)$ & $0.69^{\mathrm{F}}$ \\
\hline Epididymitis & $21(6.1 \%)$ & $21(10.2 \%)$ & NA & \\
\hline Neurological involvement & $58(16.9 \%)$ & $34(16.6 \%)$ & $24(17.4 \%)$ & 0.85 \\
\hline CNS involved & $21(6.1 \%)$ & $11(5.4 \%)$ & $10(7.2 \%)$ & 0.48 \\
\hline PNS involved & $9(2.6 \%)$ & $7(3.4 \%)$ & $2(1.4 \%)$ & $0.32^{\mathrm{F}}$ \\
\hline Vascular involvement & $71(20.7 \%)$ & $56(27.3 \%)$ & 15 (10.9\%) & $<0.001^{* * *}$ \\
\hline Venous embolism & $54(15.7 \%)$ & $44(21.5 \%)$ & $10(7.2 \%)$ & $<0.001^{* * *}$ \\
\hline Arterial embolism & $15(4.4 \%)$ & $9(4.4 \%)$ & $6(4.3 \%)$ & 0.99 \\
\hline Aneurysm & $18(5.2 \%)$ & $15(7.3 \%)$ & $3(2.2 \%)$ & $0.04^{*}$ \\
\hline
\end{tabular}

${ }^{*} P<0.05,{ }^{* *} P<0.01,{ }^{* * *} P<0.001,{ }^{\mathrm{F}}$ Fisher's exact test.

Abbreviation: PUMCH, Peking Union Medical College Hospital. CRSV, Chinese Registry for Systemic Vasculitis. ESR, erythrocyte sediment rate. CRP, c-reactive protein. VDI, vasculitis damage index. CNS, central nervous system. PNS, peripheral nervous system.

Legend: The clinical characteristics of patients with Behcet's disease(BD) registered by Peking Union Medical College Hospital (PUMCH) in Chinese Registry for Systemic Vasculitis (CRSV) database were summarized. The comparisons between male and female patients were made. Those patients were registered by PUMCH up to February 28 of 2020 
abnormalities (15.3\%), and pulmonary involvement $(9.1 \%)$ were not rare (Table 1).

Cutaneous and mucosal involvements (80.5\%) were the most common lesions in BD patients, followed by ocular involvement $(28.6 \%)$, gastrointestinal involvement $(21.6 \%)$, vascular involvement (20.7\%), and neurological involvement (16.9\%). Abnormalities of lungs $(7.3 \%)$ and heart $(7.0 \%)$ were also common (Table 2).

The involvement of ear, nose, and throat $(69.6 \%)$ was common in AAV patients, followed by involvement of lungs (46.6\%), kidneys $(46.1 \%)$, and eyes $(32.9 \%)$. The constitutional symptoms were also common, such as fatigue (50.3\%), fever $(39.8 \%)$, and weight loss $(34.0 \%)$. Cutaneous and mucosal lesions were found in $30.9 \%$ of patients. Arthralgia and/or arthritis was found in $27.2 \%$ patients, while myalgia in $22.0 \%$. Involvement of the neurological system (20.9\%) and heart (10.5\%) was not uncommon (Table 3).

Comparisons between genders of these patients were made. A significant difference in hemoglobin was found between male and female patients with TAK, BD, and AAV, physiologically (Tables 1-3). In TAK patients, higher rates of hypertension $(57.3 \%$ vs. $43.4 \%)$, stroke $(17.5 \%$ vs. $7.0 \%)$, and venous thrombosis $(9.7 \%$ vs. $4.1 \%)$ were found in male

Table 3: The clinical characteristics of patients with ANCA-associated vasculitis registered by PUMCH in CRSV

\begin{tabular}{|c|c|c|c|c|}
\hline & Total & Male & Female & $P$-value \\
\hline Patients numbers & 191 & $100(52.4 \%)$ & $91(47.6 \%)$ & \\
\hline Age at diagnosis (years) & $48.1 \pm 16.9(14.7,83.0)$ & $52.0 \pm 15.6(15.4,83.0)$ & $44.0 \pm 17.3(14.7,82.8)$ & $0.001^{* *}$ \\
\hline Age at registration (years) & $49.4 \pm 16.4(15.5,83.3)$ & $53.0 \pm 15.3(16.5,83.3)$ & $45.4 \pm 16.7(15.5,82.8)$ & $0.001 * *$ \\
\hline Disease duration before registration (years) & $1.5 \pm 2.4(0,15.0)$ & $1.3 \pm 1.8(0,12.2)$ & $1.7 \pm 2.9(0,15.0)$ & 0.30 \\
\hline \multicolumn{5}{|l|}{ Laboratory findings } \\
\hline White blood cells $\left(\times 10^{9} / \mathrm{L}\right)$ & $9.7 \pm 4.1$ & $10.1 \pm 4.0$ & $9.4 \pm 4.1$ & 0.24 \\
\hline Hemoglobin (g/L) & $129.2 \pm 20.9$ & $137.7 \pm 20.0$ & $119.2 \pm 17.3$ & $<0.001^{* * *}$ \\
\hline Platelet $\left(\times 10^{9} / \mathrm{L}\right)$ & $263.5 \pm 104.4$ & $255.5 \pm 102.9$ & $273.0 \pm 105.9$ & 0.27 \\
\hline ESR ( $1^{\text {sthour}) ~}$ & $27.8 \pm 30.3$ & $25.4 \pm 27.8$ & $30.4 \pm 32.8$ & 0.30 \\
\hline $\mathrm{CRP}(\mathrm{mg} / \mathrm{L})$ & $24.3 \pm 44.9$ & $28.5 \pm 51.1$ & $18.9 \pm 35.2$ & 0.23 \\
\hline ANCA positive & $131(68.6 \%)$ & 71 (71.0\%) & $60(65.9 \%)$ & 0.45 \\
\hline c-ANCA positive & 38 (19.9\%) & $22(22.0 \%)$ & $16(17.6 \%)$ & 0.45 \\
\hline p-ANCA positive & $44(23.0 \%)$ & $18(18.0 \%)$ & $26(28.6 \%)$ & 0.08 \\
\hline anti-PR3-ANCA positive & 61 (31.9\%) & $41(41.0 \%)$ & $20(22.0 \%)$ & $<0.01^{* *}$ \\
\hline anti-MPO-ANCA positive & 65 (34.0\%) & $27(27.0 \%)$ & $38(41.8 \%)$ & $0.03^{*}$ \\
\hline \multicolumn{5}{|l|}{ Clinical evaluations } \\
\hline BVAS & $9.2 \pm 6.8(0,36)$ & $8.4 \pm 6.5(0,28)$ & $10.1 \pm 7.1(0,36)$ & 0.15 \\
\hline VDI & $2.1 \pm 1.6(0,7)$ & $2.2 \pm 1.6(0,7)$ & $2.0 \pm 1.6(0,6)$ & 0.41 \\
\hline AVID & $2.4 \pm 1.8(0,7)$ & $2.4 \pm 1.8(0,7)$ & $2.4 \pm 1.7(0,6)$ & 0.79 \\
\hline \multicolumn{5}{|l|}{ Clinical manifestations } \\
\hline Fatigue & $96(50.3 \%)$ & $54(54.0 \%)$ & $42(46.2 \%)$ & 0.28 \\
\hline Fever (over 38 centigrade) & $76(39.8 \%)$ & 47 (47.0\%) & 29 (31.9\%) & $0.03 *$ \\
\hline Weight loss & 65 (34.0\%) & 35 (35.0\%) & $30(33.0 \%)$ & 0.77 \\
\hline Arthralgia/arthritis & $52(27.2 \%)$ & $29(29.0 \%)$ & $23(25.3 \%)$ & 0.56 \\
\hline myalgia & $42(22.0 \%)$ & $24(24.0 \%)$ & $18(19.8 \%)$ & 0.48 \\
\hline Involvement of ear, nose and throat & $133(69.6 \%)$ & $71(71.0 \%)$ & $62(68.1 \%)$ & 0.67 \\
\hline Nasal blockage/chronic discharge/crusting & $82(42.9 \%)$ & $44(44.0 \%)$ & $38(41.8 \%)$ & 0.76 \\
\hline Sinusitis & $76(39.8 \%)$ & $41(41.0 \%)$ & $35(38.5 \%)$ & 0.72 \\
\hline Crusting/nasal ulcer & $69(36.1 \%)$ & $37(37.0 \%)$ & $32(35.2 \%)$ & 0.79 \\
\hline Bloody nasal discharge & $53(27.7 \%)$ & $29(29.0 \%)$ & $24(26.4)$ & 0.69 \\
\hline Hearing loss & 49 (25.7\%) & $27(27.0 \%)$ & $22(24.2 \%)$ & 0.66 \\
\hline Hoarseness & $36(18.8 \%)$ & $19(19.0 \%)$ & $17(18.7 \%)$ & 0.96 \\
\hline Nasal bridge collapse & $26(13.6 \%)$ & $12(12.0 \%)$ & $14(15.4 \%)$ & 0.50 \\
\hline
\end{tabular}


Table 3: Continued

\begin{tabular}{|c|c|c|c|c|}
\hline & Total & Male & Female & $P$-value \\
\hline Subglottic stenosis & $16(8.4 \%)$ & $3(3.0 \%)$ & $13(14.3 \%)$ & $<0.01^{* *}$ \\
\hline Septal perforation & $11(5.8 \%)$ & $7(7.0 \%)$ & $4(4.4 \%)$ & 0.44 \\
\hline Nasal polyps & $10(5.2 \%)$ & $5(5.0 \%)$ & $5(5.5 \%)$ & $1.00^{\mathrm{F}}$ \\
\hline Pulmonary abnormalities & $89(46.6 \%)$ & $49(49.0 \%)$ & $40(44.0 \%)$ & 0.49 \\
\hline Nodules/cavity & $45(23.6 \%)$ & $23(23.0 \%)$ & $22(24.2 \%)$ & 0.85 \\
\hline Hemoptysis & $24(12.6 \%)$ & $11(11.0 \%)$ & $13(14.3 \%)$ & 0.49 \\
\hline Pleurisy/pleural effusion & $17(8.9 \%)$ & $11(11.0 \%)$ & $6(6.6 \%)$ & 0.29 \\
\hline Renal abnormalities & $88(46.1 \%)$ & $45(45.0 \%)$ & $43(47.3 \%)$ & 0.76 \\
\hline Proteinuria ( $>0.2 \mathrm{~g} / 24$ hours) & $72(37.7 \%)$ & $38(38.0 \%)$ & $34(37.4 \%)$ & 0.93 \\
\hline Hematuria & $42(22.0 \%)$ & $22(22.0 \%)$ & $20(22.0 \%)$ & 1.00 \\
\hline Increased serum level of creatinine & $34(17.8 \%)$ & $20(20.0 \%)$ & $14(15.4 \%)$ & 0.41 \\
\hline Ocular involvement & $63(32.9 \%)$ & $37(37.0 \%)$ & $26(28.6 \%)$ & 0.22 \\
\hline Blurred vision & 37 (19.4\%) & $21(21.0 \%)$ & $16(17.6 \%)$ & 0.55 \\
\hline Conjunctivitis/episcleritis & $24(12.6 \%)$ & $13(13.0 \%)$ & $11(12.1 \%)$ & 0.85 \\
\hline Diplopia & $22(11.5 \%)$ & $15(15.0 \%)$ & $7(7.7 \%)$ & 0.11 \\
\hline Ophthalmodynia & $18(9.4 \%)$ & $9(9.0 \%)$ & $9(9.9 \%)$ & 0.83 \\
\hline Exophthalmos & $15(7.9 \%)$ & $8(8.0 \%)$ & $7(7.7 \%)$ & 0.94 \\
\hline Scleritis & $13(6.8 \%)$ & $5(8.0 \%)$ & $8(8.8 \%)$ & 0.30 \\
\hline Cutaneous and mucosal lesions & $59(30.9 \%)$ & $33(33.0 \%)$ & $26(28.6 \%)$ & 0.51 \\
\hline Oral ulcer & $27(14.1 \%)$ & $16(16.0 \%)$ & $11(12.1 \%)$ & 0.44 \\
\hline Purpura & $15(7.9 \%)$ & $6(6.0 \%)$ & $9(9.9 \%)$ & 0.32 \\
\hline Neurological involvement & $40(20.9 \%)$ & $29(29.0 \%)$ & $11(12.1 \%)$ & $<0.01^{* *}$ \\
\hline CNS involved & $12(6.3 \%)$ & $9(9.0 \%)$ & $3(3.3 \%)$ & 0.11 \\
\hline PNS involved & $29(15.2 \%)$ & 19 (19.0\%) & $10(11.0 \%)$ & 0.12 \\
\hline Motor polymononeuritis & $23(12.0 \%)$ & $16(16.0 \%)$ & $7(7.7 \%)$ & 0.08 \\
\hline Sensory peripheral neuropathy & $13(6.8 \%)$ & $7(7.0 \%)$ & $6(6.6 \%)$ & 0.91 \\
\hline Abnormalities of heart & $20(10.5 \%)$ & $10(10.0 \%)$ & $10(11.0 \%)$ & 0.82 \\
\hline Collapse of vertebra & $16(8.4 \%)$ & $9(9.0 \%)$ & $7(7.7 \%)$ & 0.75 \\
\hline Aseptic necrosis & $9(4.7 \%)$ & $5(5.0 \%)$ & $4(4.4 \%)$ & $1.00^{\mathrm{F}}$ \\
\hline
\end{tabular}

${ }^{*} P<0.05,{ }^{* *} P<0.01,{ }^{* * *} P<0.001,{ }^{\mathrm{F}}$ Fisher's exact test.

Abbreviation: AAV, ANCA-associated vasculitis. ANCA, anti-neutrophil cytoplasm antibody. c-ANCA, cytoplasmic ANCA. p-ANCA, perineuclear ANCA. anti-PR3, anti-proteinase 3. Anti-MPO, anti-myeloperoxidase. PUMCH, Peking Union Medical College Hospital. CRSV, Chinese Registry for Systemic Vasculitis. ESR, erythrocyte sediment rate. CRP, c-reactive protein. BVAS, Birmingham vasculitis activity score. VDI, vasculitis damage index. AVID, ANCA-associated vasculitis index of damage. CNS, central nervous system. PNS, peripheral nervous system.

Legend: The clinical characteristics of patients with ANCA-associated arteritis(AAV) from Peking Union Medical College Hospital (PUMCH) cohorts in Chinese Registry for Systemic Vasculitis (CRSV) database were summarized. The comparisons between male and female patients were made. Those patients were registered by PUMCH up to February 28 of 2020.

patients, and higher disease activity (Birmingham vasculitis activity score [BVAS] 9.8 vs. 8.4), higher rates of vessel bruits in neck ( $76.4 \%$ vs. $53.4 \%)$, asymmetric blood pressure in bilateral limbs $(63.8 \%$ vs. $50.5 \%)$, intermittent limbs claudication $(47.8 \%$ vs. $35.0 \%)$, carotidynia $(28.2 \%$ vs. $17.5 \%)$, headache $(36.8 \%$ vs. $26.2 \%)$, dizziness ( $38.9 \%$ vs. $28.2 \%)$, ocular involvement ( $16.7 \%$ vs. $6.8 \%)$, and amaurosis $(12.6 \%$ vs. $5.8 \%$ ) were found in female patients (Table 1). In BD patients, higher peripheral blood white blood cells count $(8.5$ vs. $7.3 \times 10 \%$ ), higher rates of pseudofolliculitis $(20.5 \%$ vs. $10.1 \%)$, acne-like rash ( $11.7 \%$ vs. $4.3 \%)$, blurred vision
( $24.4 \%$ vs. $13.8 \%)$, uveitis ( $22.9 \%$ vs. $11.6 \%)$, cataract $(5.9 \%$ vs. $1.4 \%)$, vascular involvement $(27.3 \%$ vs. $10.9 \%)$, including venous embolism $(21.5 \%$ vs. $7.2 \%)$ and aneurysm $(7.3 \%$ vs. $2.2 \%$ ), were found in male patients, with higher rate of genital ulcer $(70.3 \%$ vs. $50.7 \%$ ) in females (Table 2 ). In AAV patients, higher rate of fever $(47.0 \%$ vs. $31.9 \%)$ and neurological involvement $(29.0 \%$ vs. $12.1 \%)$, as well as higher positive rates of PR3-ANCA ( $41.0 \%$ vs. $22.0 \%$ ), were found in male patients, and younger age at diagnosis (44.0 years vs. 52.0 years) and higher positive rate of MPO-ANCA (41.8\% vs. $27.0 \%$ ) were found in females (Table 3 ). 
In 31 patients with the definite diagnosis of PAN in the CRSVPUMCH cohort, only 1 patient had evidence of hepatitis B virus (HBV) infection, and 4 patients had evidence for history of HBV infection. The clinical features of these patients are summarized in Table 4. The clinical manifestations of 53 patients with USV are summarized in Table 5.

In addition to the major clinical characteristics, specific minor features were further investigated in our cohort and are summarized in Tables $1-5$.

Table 4: The clinical characteristics of patients with PAN registered by $\mathrm{PUMCH}$ in $\mathrm{CRSV}$

\begin{tabular}{|c|c|}
\hline & PAN ( $N=31)$ \\
\hline Male patients number, $\mathrm{n}(\%)$ & $20(64.5 \%)$ \\
\hline Age at diagnosis (years) & $38.6 \pm 14.4(14.8,71.1)$ \\
\hline Age at registration (years) & $41.0 \pm 15.1(15.7,71.3)$ \\
\hline Disease duration before registration (years) & $0.9 \pm 1.3(0,5.6)$ \\
\hline \multicolumn{2}{|l|}{ Laboratory findings } \\
\hline White blood cells $\left(\times 10^{9} / \mathrm{L}\right)$ & $9.5 \pm 3.9$ \\
\hline Hemoglobin (g/L) & $138.4 \pm 20.8$ \\
\hline Platelet $\left(\times 10^{9} / \mathrm{L}\right)$ & $293.9 \pm 97.6$ \\
\hline ESR ( $1^{\text {sthour }}$ ) & $22.3 \pm 29.0$ \\
\hline $\mathrm{CRP}(\mathrm{mg} / \mathrm{L})$ & $13.0 \pm 18.3$ \\
\hline \multicolumn{2}{|l|}{ Clinical evaluations } \\
\hline BVAS & $4.9 \pm 6.8(0,27)$ \\
\hline VDI & $1.8 \pm 1.8(0,8)$ \\
\hline \multicolumn{2}{|l|}{ Clinical manifestations } \\
\hline Fatigue & $12(38.7 \%)$ \\
\hline Fever (over 38 centigrade) & $10(32.3 \%)$ \\
\hline Weight loss & $9(29.0 \%)$ \\
\hline Arthralgia/arthritis & $11(35.5 \%)$ \\
\hline myalgia & $16(51.6 \%)$ \\
\hline Pulmonary abnormalities & $1(3.2 \%)$ \\
\hline Nodules/cavity & $1(3.2 \%)$ \\
\hline Wheeze & $1(3.2 \%)$ \\
\hline Renal abnormalities & $3(9.7 \%)$ \\
\hline Proteinuria ( $>0.2 \mathrm{~g} / 24$ hours) & $1(3.2 \%)$ \\
\hline Hematuria & $2(6.5 \%)$ \\
\hline Increased serum level of creatinine & $1(3.2 \%)$ \\
\hline Ocular involvement & $5(16.1 \%)$ \\
\hline Blurred vision & $2(6.5 \%)$ \\
\hline Conjunctivitis/episcleritis & $2(6.5 \%)$ \\
\hline Exophthalmos & $2(6.5 \%)$ \\
\hline Cutaneous and mucosal lesions & 19 (61.3\%) \\
\hline Oral ulcer & $3(9.7 \%)$ \\
\hline Livedo reticularis & $7(22.6 \%)$ \\
\hline Skin ulcer & $7(22.6 \%)$ \\
\hline Gangrene & $5(16.1 \%)$ \\
\hline
\end{tabular}

Table 4: Continued

\begin{tabular}{lc}
\hline & PAN (N=31) \\
\hline Purpura & $3(9.7 \%)$ \\
Raynaud's phenomenon & $2(6.5 \%)$ \\
Neurological involvement & $10(32.3 \%)$ \\
CNS involved & $2(6.5 \%)$ \\
Stroke & $3(9.7 \%)$ \\
PNS involved & $8(25.8 \%)$ \\
Motor polymononeuritis & $2(6.5 \%)$ \\
Sensory peripheral neuropathy & $1(3.2 \%)$ \\
Disappeared peripheral arterial pulsation & $2(6.5 \%)$ \\
Intermittent claudication of limbs & $7(22.6 \%)$ \\
Myocardial infarction & $1(3.2 \%)$ \\
Angioplasty & $2(6.5 \%)$ \\
fragile fracture & $1(3.2 \%)$ \\
Aseptic necrosis & $2(6.5 \%)$ \\
\hline
\end{tabular}

PAN, polyarteritis nodosa. PUMCH: Peking Union Medical College Hospital. CRSV: Chinese Registry for Systemic Vasculitis.

Legend: The clinical characteristics of patients with polyarteritis nodosa (PAN) were summarized. Due to the limited number of patients, comparison between genders was not performed. Those patients were registered by PUMCH up to February 28 of 2020 .

Table 5: The clinical characteristics of patients with USV registered by PUMCH in CRSV

\begin{tabular}{lc}
\hline & USV (N=53) \\
\hline Male, $\mathrm{n}$ (\%) & $24(45.3 \%)$ \\
Age at diagnosis (years) & $39.4 \pm 15.9(14.7,86.9)$ \\
Age at registration (years) & $40.6 \pm 14.9(15.5,87.0)$ \\
Disease duration before registration (years) & $1.2 \pm 2.1(0,9.3)$ \\
Laboratory findings & \\
White blood cells (×10\%/L) & $8.2 \pm 2.8$ \\
Hemoglobin (g/L) & $132.8 \pm 23.8$ \\
Platelet (×10 $/ \mathrm{L})$ & $274.2 \pm 104.8$ \\
ESR (15thour) & $27.4 \pm 31.8$ \\
CRP (mg/L) & $19.7 \pm 30.3$ \\
Clinical evaluations & \\
BVAS & $2.8 \pm 2.9(0,10)$ \\
VDI & $1.6 \pm 1.1(0,5)$ \\
Clinical manifestations & \\
Fatigue & $24(45.3 \%)$ \\
Fever (over 38 centigrade) & $11(20.8 \%)$ \\
Weight loss & $14(26.4 \%)$ \\
Arthralgia/arthritis & $15(28.3 \%)$ \\
myalgia & $12(22.6 \%)$ \\
Involvement of ear, nose and throat & $15(28.3 \%)$ \\
Nasal blockage/chronic discharge/crusting & $3(5.7 \%)$ \\
Sinusitis & $2(3.8 \%)$ \\
Crusting/nasal ulcer & $37(37.0 \%)$ \\
\hline & \\
& \\
&
\end{tabular}


Table 5: Continued

\begin{tabular}{|c|c|}
\hline & USV $(\mathrm{N}=53)$ \\
\hline Bloody nasal discharge & $2(3.8 \%)$ \\
\hline Hearing loss & $2(3.8 \%)$ \\
\hline Hoarseness & $6(11.3 \%)$ \\
\hline Nasal bridge collapse & $2(3.8 \%)$ \\
\hline Subglottic stenosis & $4(7.5 \%)$ \\
\hline Auricular cartilage involvement & $3(5.7 \%)$ \\
\hline Pulmonary abnormalities & $8(15.1 \%)$ \\
\hline Nodules/cavity & $3(5.7 \%)$ \\
\hline Hemoptysis & $1(1.9 \%)$ \\
\hline Pleurisy/pleural effusion & $4(7.5 \%)$ \\
\hline Renal abnormalities & $2(3.8 \%)$ \\
\hline Proteinuria ( $>0.2 \mathrm{~g} / 24$ hours) & $2(3.8 \%)$ \\
\hline Hematuria & $1(1.9 \%)$ \\
\hline Increased serum level of creatinine & $1(1.9 \%)$ \\
\hline Ocular involvement & $9(17.0 \%)$ \\
\hline Blurred vision & $6(11.3 \%)$ \\
\hline Amaurosis & $2(3.8 \%)$ \\
\hline Conjunctivitis/episcleritis & $2(3.8 \%)$ \\
\hline Diplopia & $1(1.9 \%)$ \\
\hline Ophthalmodynia & $2(3.8 \%)$ \\
\hline Scleritis & $1(1.9 \%)$ \\
\hline Uveitis & $3(5.7 \%)$ \\
\hline Cutaneous and mucosal lesions & $14(26.4 \%)$ \\
\hline Oral ulcer & $9(17.0 \%)$ \\
\hline Livedo reticularis & $1(1.9 \%)$ \\
\hline Skin ulcer & $5(9.4 \%)$ \\
\hline Gangrene & $2(3.8 \%)$ \\
\hline Purpura & $3(5.7 \%)$ \\
\hline Raynaud's phenomenon & $1(1.9 \%)$ \\
\hline Gingivitis & $7(13.2 \%)$ \\
\hline Neurological involvement & $14(26.4 \%)$ \\
\hline CNS involved & $7(13.2 \%)$ \\
\hline Stroke & $6(11.3 \%)$ \\
\hline PNS involved & $9(17.0 \%)$ \\
\hline Motor polymononeuritis & $2(3.8 \%)$ \\
\hline Sensory peripheral neuropathy & $1(1.9 \%)$ \\
\hline Intermittent claudication of limbs & $3(5.7 \%)$ \\
\hline Abnormalities of heart & $5(9.4 \%)$ \\
\hline Myocardial infarction & $1(1.9 \%)$ \\
\hline Valvar abnormalities & $3(5.7 \%)$ \\
\hline Gastrointestinal abnormalities & $2(3.8 \%)$ \\
\hline Mesangial ischemia & $1(1.9 \%)$ \\
\hline Intestinal perforation & $1(1.9 \%)$ \\
\hline Angioplasty & $4(7.5 \%)$ \\
\hline Aseptic necrosis & $2(3.8 \%)$ \\
\hline
\end{tabular}

USV, unclassified systemic vasculitis. PUMCH: Peking Union Medical College Hospital. CRSV: Chinese Registry for Systemic Vasculitis.

Legend: The clinical characteristics of patients with unclassified systemic

vasculitis (USV) were summarized. Due to the limited number of patients, comparison between genders was not performed. Those patients were registered by PUMCH up to February 28 of 2020.

\section{Discussion}

Several registries for patients with vasculitis have been developed by different countries. The best known registry database and patient cohort for systemic vasculitis are the Vasculitis Clinical Research Consortium (VCRC) in the United States, ${ }^{[9]}$ and the French vasculitis study group (FVSG) developed by French rheumatologists, which mainly included patients with PAN and AAV. ${ }^{[10,11]}$ There are also several databases for systemic vasculitis in Europe, Canada, and Asia. All these registries for systemic vasculitis have made important contributions to further the understanding of the nature and characteristics of systemic vasculitis. Based on these patient cohorts and registry databases, we achieved several milestones in the history of vasculitis study such as the Chapel Hill consensus on vasculitis classification and nomenclature ${ }^{[6]}$ five-factor score (FFS) for outcome evaluation, BVAS for disease activity assessment, ${ }^{[12,13]}$ particularly for AAV, and the Diagnosis and Classification of Vasculitis Study (DCVAS) study. ${ }^{[14]}$ From these databases, we know that there are ethnical differences in the clinical spectrum of systemic vasculitis.

The CRSV is the only nationwide registry for systemic vasculitis in China and is also the biggest one in the world, according to our knowledge. From 2018 to 2020, the numbers of registered patients had doubled, which demonstrated that the application of the CRSV had accelerated in these recent 2 years. As the leading unit, $\mathrm{PUMCH}$ had registered about one-third patients of the CRSV. This registry not only provides valuable information about the clinical features and prognosis of Chinese patients with vasculitis but also provides the infrastructure for conducting clinical research. We carried out several studies with the support of the CRSV, including observational studies and drug studies. In this study, we analyzed the major clinical manifestations of several types of vasculitis, and compared the clinical profiles of these vasculitis based on age and gender.

In our TAK cohort, vascular abnormalities_-including physical findings of vessel bruits, asymmetric blood pressure in bilateral limbs, limbs claudication, and carotidynia-were the most common clinical findings, and the constitutional symptoms were also common, such as fever and weight loss. Among the internal organs involved, heart and kidney were the most common. Symptoms related with nervous system involvement, including headache, dizziness, and syncope were more frequently found in female patients, while stroke was more common in males. Cutaneous and mucosal lesions, as well as ocular abnormalities, were not rare in TAK patients. In addition, pulmonary involvement was found in 9.1\% patients with TAK, including pulmonary infiltration and abnormalities of pulmonary artery (wall thickness, stenosis, dilation, occlusion/infarction, and hypertension). More than one-fourth of patients $(27 \%)$ were treated with angioplasty 
or stent implantation or open surgery, which was close to other cohorts (one-third). ${ }^{[15]}$

BD patients are the second largest patient population in our cohort (Figure 2). Cutaneous and mucosal involvements were the most common lesions, followed by ocular, gastrointestinal, vascular, and neurological involvements. Ocular involvement (including blurred vision, uveitis, and cataract) and vascular involvement (especially venous embolism and aneurysm) were more commonly found in male patients than in female BD patients, which was similar to other cohorts reported in the literature. ${ }^{[16]}$

The possible reason for the relatively small proportion of AAV registered might be that some AAV patients visited nephrologists or pulmonary physicians because of the most prominently involved organ being kidney and lung in China. A similar situation was found while comparing the study populations of AAV patients between different databases, such as VCRC and the European Vasculitis Society (EUVAS). ${ }^{[9]}$ The most commonly involved organs in our cohort were ear, nose, and throat, followed by the lungs and kidneys. Comparisons of clinical manifestations between genders revealed that the frequency of fever and neurological involvement in male patients with $\mathrm{AAV}$ was higher than in females. Both the peripheral and central nerve systems were involved in AAV patients. This was quite different from patients with TAK, in which only central nerve system involvement was observed, while no evidence of peripheral nerve system involvement was found.

We only had 31 patients with a definite diagnosis of PAN. The major reason is the wide use of HBV vaccination in China; consequentially, HBV-infection-related PAN has become very rare in clinical practice. The majority of our PAN patients are non-HBV-related.

The analysis of the demographic characteristics of patients with these 5 systemic vasculitis have shown that $B D, A A V$, and PAN are more prevalent in male patients than in females, while the situation of TAK patients is on the contrary. This is similar to the descriptions in the review by Gonzalez-Gay et al., ${ }^{[17]}$ although the number of patients with PAN is very limited in the CRSV. The age at disease diagnosis is also different among these 5 vasculitis diseases. The average age at diagnosis is 29.6 years in TAK patients, while it is 48.1 years in AAV patients. In patients with $\mathrm{AAV}$, age at diagnosis and registration of female patients are younger than in males in our cohort, but we failed to find similar results in other cohort reported in the literature. ${ }^{[18-21]}$ However, this needs to be confirmed by large sample studies, as opposed to the limited sample size of our cohort.

The strength of this study is that all the information is derived from the largest database of systemic vasculitis in the country. There are some limitations in this study. $\mathrm{PUMCH}$ is a national referral center of China; thus, disease severity of patients of PUMCH may be more severe than patients from other hospitals in the country. Another limitation is that some AAV patients with predominant renal or pulmonary involvement may not come to the rheumatology department, and so the clinical presentation of AAV patients of the registry may be biased.

\section{Conclusion}

The clinical features of Chinese patients with systemic vasculitis are different from other reported cohort studies, based on the data from the CRSV, which is the first registry database for systemic vasculitis by rheumatologists in China.

Ethics Approval and Consent to Participate

This study was approved by Institutional Review Board of Peking Union Medical College Hospital, Beijing, China (S-478). Written informed consent was obtained from all participants and the study was performed in accordance with the Declaration of Helsinki. Personal information was protected and kept anonymous in the Chinese Registry of Systemic Vasculitis (CRSV) database.

Availability of Data and Material

The datasets used and/or analyzed during the current study are available from the corresponding author on reasonable request.

Conflict of Interest

Xiaofeng Zeng is the Editor-in-Chief of the journal, Xinping Tian is the executive Editor-in-Chief, and Mengtao Li is an Associate Editor-inChief. The article was subject to the journal's standard procedures, with peer review handled independently of these members and their research groups. 
Funding

This work was supported by the National Clinical Research Center for Dermatologic and Immunologic Diseases (NCRC-DID), the Chinese National Key Research and Development Program (Grant nos. 2017YFC0907600, 2008BAI59B02), and the Chinese National High Technology Research and Development Program, Ministry of Science and Technology (Grant number 2012AA02A513).

\section{Authors' Contributions}

$X T$ and $X Z$ conceived and designed the study. ML advised on the design of the study. JL, WZ, and YY collected the data. JL, JZ, and YW analyzed and interpreted the patient data. $J L$ and $X T$ were major contributors in writing the manuscript. JL, $X T$, and $X Z$ edited and revised the paper. All authors read and approved the final manuscript.

Acknowledgment

Not applicable.

\section{References}

[1] Li M, Tian X, Zhang W, Leng X, Zeng X. CRDC: A Chinese Rheumatology Research Platform. Clin Rheumatol. 2015;34(8): 1347-1352.

[2] Arend WP, Michel BA, Bloch DA, et al. The American College of Rheumatology 1990 Criteria for the Classification of Takayasu Arteritis. Arthritis Rheum. 1990;33(8):1129-1134.

[3] Lightfoot RW, Jr., Michel BA, Bloch DA, et al. The American College of Rheumatology 1990 Criteria for the Classification of Polyarteritis Nodosa. Arthritis Rheum. 1990;33(8):1088-1093.

[4] Leavitt RY, Fauci AS, Bloch DA, et al. The American College of Rheumatology 1990 Criteria for the Classification of Wegener's Granulomatosis. Arthritis Rheum. 1990;33(8):1101-1107.

[5] Masi AT, Hunder GG, Lie JT, et al. The American College of Rheumatology 1990 Criteria for the Classification of Churg-Strauss Syndrome (Allergic Granulomatosis and Angiitis). Arthritis Rheum. 1990;33(8):1094-1100.

[6] Jennette JC, Falk RJ, Bacon PA, et al. 2012 Revised International Chapel Hill Consensus Conference Nomenclature of Vasculitides. Arthritis Rheum. 2013;65(1):1-11

[7] The International Criteria for Behçet's Disease (ICBD): A Collaborative Study of 27 Countries on the Sensitivity and Specificity of the New Criteria. J Eur Acad Dermatol Venereol. 2014;28(3):338-347.

[8] Hunder GG, Bloch DA, Michel BA, et al. The American College of Rheumatology 1990 Criteria for the Classification of Giant Cell Arteritis. Arthritis Rheum. 1990;33(8):1122-1128.

[9] Pagnoux C, Carette S, Khalidi NA, et al. Comparability of Patients with ANCA-Associated Vasculitis Enrolled in Clinical Trials or in Observational Cohorts. Clin Exp Rheumatol. 2015;33(2 Suppl 89):S-77-S-83.

[10] Guillevin L, Lhote F, Gayraud M, et al. Prognostic Factors in Polyarteritis Nodosa and Churg-Strauss Syndrome. A Prospective Study in 342 Patients. Medicine (Baltimore). 1996;75(1):17-28.

[11] Guillevin L, Pagnoux C, Seror R, Mahr A, Mouthon L, Le Toumelin P. The Five-Factor Score Revisited: Assessment of Prognoses of Systemic Necrotizing Vasculitides Based on the
French Vasculitis Study Group (FVSG) Cohort. Medicine (Baltimore). 2011;90(1):19-27.

[12] Luqmani RA, Bacon PA, Moots RJ, et al. Birmingham Vasculitis Activity Score (BVAS) in Systemic Necrotizing Vasculitis. QJM. 1994;87(11):671-678.

[13] Mukhtyar C, Lee R, Brown D, et al. Modification and Validation of the Birmingham Vasculitis Activity Score (Version 3). Ann Rheum Dis. 2009;68(12):1827-1832.

[14] Luqmani RA, Suppiah R, Grayson PC, Merkel PA, Watts R. Nomenclature and Classification of Vasculitis - Update on the ACR/ EULAR Diagnosis and Classification of Vasculitis Study (DCVAS). Clin Exp Immunol. 2011;164(Suppl 1):11-13.

[15] Mason JC. Surgical Intervention and its Role in Takayasu Arteritis. Best Pract Res Clin Rheumatol. 2018;32(1):112-124.

[16] Bonitsis NG, Luong Nguyen LB, LaValley MP, et al. GenderSpecific Differences in Adamantiades-Behcet's Disease Manifestations: An Analysis of the German Registry and Meta-Analysis of Data from the Literature. Rheumatology (Oxford, England). 2015;54(1):121-133.

[17] Gonzalez-Gay MA, Garcia-Porrua C. Epidemiology of the Vasculitides. Rheum Dis Clin North Am. 2001;27(4):729-749.

[18] Flossmann O, Berden A, de Groot $\mathrm{K}$, et al. Long-Term Patient Survival in ANCA-Associated Vasculitis. Ann Rheum Dis. 2011;70(3):488-494.

[19] Heijl C, Mohammad AJ, Westman K, Hoglund P. Long-Term Patient Survival in A Swedish Population-Based Cohort of Patients with ANCA-Associated Vasculitis. RMD Open. 2017;3(1):e000435.

[20] Hilhorst $M$, Wilde $B$, van Paassen $P$, Winkens $B$, van Breda Vriesman $P$, Cohen Tervaert JW. Improved Outcome in Anti-Neutrophil Cytoplasmic Antibody (ANCA)-Associated Glomerulonephritis: A 30-Year Follow-Up Study. Nephrol Dial Transplant. 2013;28(2):373-379. [21] Mohammad AJ, Jacobsson LT, Mahr AD, Sturfelt G, Segelmark M. Prevalence of Wegener's Granulomatosis, Microscopic Polyangiitis, Polyarteritis Nodosa and Churg-Strauss Syndrome within A Defined Population in Southern Sweden. Rheumatology (Oxford). 2007;46(8):1329-1337. 